\title{
Cortical excitability and sleep deprivation: a transcranial magnetic stimulation study
}

\author{
C Civardi, C Boccagni, R Vicentini, L Bolamperti, R Tarletti, C Varrasi, F Monaco, \\ R Cantello
}

\begin{abstract}
The objective was to assess the changes in cortical excitability after sleep deprivation in normal subjects. Sleep deprivation activates EEG epileptiform activity in an unknown way. Transcranial magnetic stimulation (TMS) can inform on the excitability of the primary motor cortex. Eight healthy subjects (four men and four women) were studied. Transcranial magnetic stimulation (single and paired) was performed by a focal coil over the primary motor cortex, at the "hot spot" for the right first dorsal interosseous muscle. The following motor evoked potential features were measured: $(a)$ active and resting threshold to stimulation; (b) duration of the silent period; (c) amount of intracortical inhibition on paired TMS at the interstimulus intervals of 2 and $3 \mathrm{~ms}$ and amount of facilitation at interstimulus intervals of 14 and 16 ms. The whole TMS session was repeated after a sleep deprivation of at least 24 hours. After the sleep deprivation, the threshold to stimulation (in the active and resting muscle), as well as the silent period, did not change significantly. By contrast, the paired stimulus study showed a significant $(p<0.05)$ reduction in both intracortical inhibition and facilitation. Thus, TMS showed that sleep deprivation is associated with changes in inhibitionfacilitation balance in the primary motor cortex of normal subjects. These changes might have a link with the background factors of the "activating" effects of sleep deprivation.

(F Neurol Neurosurg Psychiatry 2001;71:809-812)
\end{abstract}

Keywords: sleep deprivation; cortical excitability; transcranial magnetic stimulation

Sleep deprivation is a powerful activator of seizures in nearly all types of epilepsy. ${ }^{1}$ It is also the best method for provoking EEG epileptiform abnormalities, or enhancing those induced by intermittent light stimulation and hyperventilation. ${ }^{2}$ Few workers ${ }^{3}$ attributed the epileptic activation to drowsiness alone, and most concluded that sleep deprivation has a specific activating effect on its own. ${ }^{4}$ However, its intrinsic mechanisms are still obscure. In animals, sleep deprivation results in a lowering of the thresholds to electroshock convulsions ${ }^{5}$ and for kindling to occur. ${ }^{6}$ This could relate to a change in the balance between excitatory and inhibitory neurotransmitters. ${ }^{7}$

Transcranial magnetic stimulation (TMS) is a safe probe of neuronal excitability of the primary motor cortex. Several variables contribute to assessment, of which the threshold to stimulation and the silent period may be assessed by the single pulse technique. Then, the paired pulse method allows measurement of the so called intracortical (corticocortical) inhibition and facilitation curve. ${ }^{8}$ This describes the effects that a weak (submotor threshold) conditioning magnetic shock induces on a test stimulus set to produce a motor evoked potential (MEP) of $0.5-1 \mathrm{mV}$. Intracortical inhibition and facilitation are thought to reflect the excitability of separate populations of interneurons intrinsic to cortical area $4 .^{9}$ Thus, we used these TMS methods to detect possible changes in the cortical physiology of normal subjects induced by a sleep deprivation of at least 24 hours.

\section{Subjects and methods}

Eight healthy volunteers (four men and four women, mean age 28.7 (SD 4.2) years; range 25 to 36 years) were studied. All gave their informed consent. The local ethics committee approved the experimental procedures. Awake subjects sat in a comfortable chair with their eyes open. Two monophasic electromagnetic stimulators (Magstim 200, Magstim Co, Whitland, Dyfed, UK) were used coupled with a Bistim device. The TMS was performed with a "figure of eight" or "butterfly" coil, delivering focal pulses over the left primary motor cortex, at the "hot spot" for the right first dorsal interosseous muscle. Motor evoked potentials (MEPs) were recorded from this muscle via surface $\mathrm{Ag}-\mathrm{AgCl}$ cup electrodes (diameter=9 $\mathrm{mm}$ ). A Viking 4 machine (Nicolet Biomedical, Madison, WI, USA) amplified $(0.1-5 \mathrm{mV} / \mathrm{cm})$ and filtered $(20-5000 \mathrm{~Hz})$ the signal, then stored it on hard disks. The sampling rate for digitisation was $25 \mathrm{kHz}$. Firstly, the following variables were determined with a single stimulator: (1) relaxed threshold (rT), defined as the minimum stimulator intensity that evoked at least $50 \%$ of responses with an amplitude of 50 $\mu \mathrm{V}$ or more (sensitivity $0.1 \mathrm{mV} /$ division, 
A

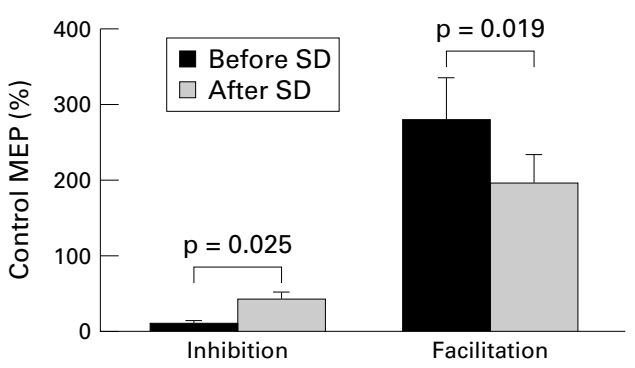

B

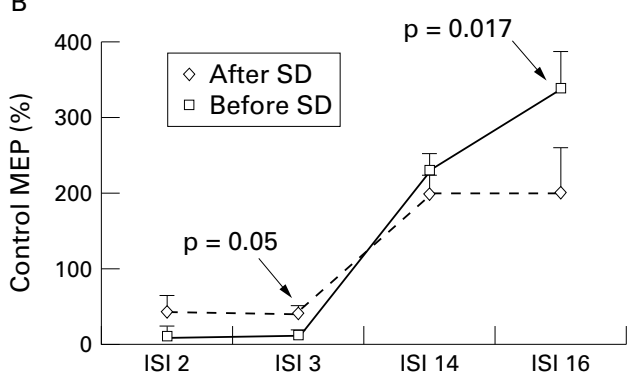

C
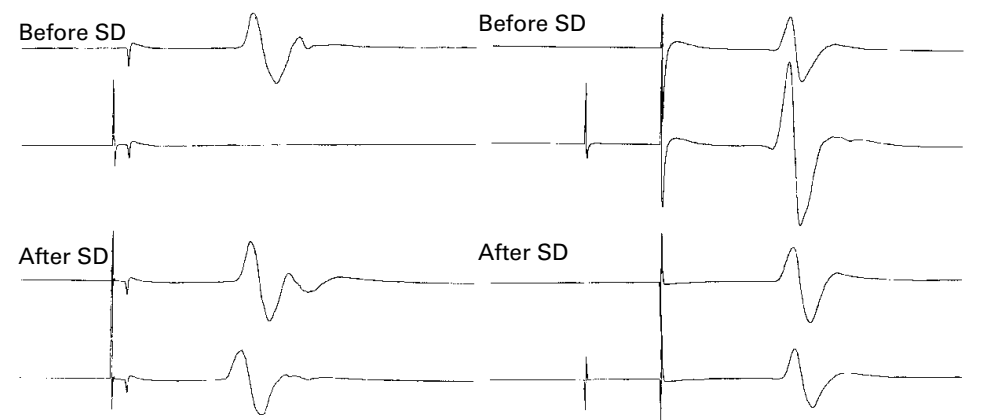

ISI $3 \mathrm{~ms}$

ISI $16 \mathrm{~ms}$

$10 \mathrm{~ms}$

$0.25 \mathrm{mV}$

Figure 1 Sleep deprivation: results on paired pulse transcranial magnetic stimulation. (A) Comparison of the inhibitory and facilitatory effects. The results for ISIs 2 and $3 \mathrm{~ms}$, then for ISIs 14 and $16 \mathrm{~ms}$ were averaged. A significant reduction of both inhibition ( $p=0.025)$ and facilitation $(p=0.019)$ after sleep deprivation was found. Black column=before sleep deprivation (SD); grey column=after SD; bars=standard deviation. (B) Effects of sleep deprivation on the single ISIs of the inhibition and facilitation curve. Squares=before $S D$ diamonds=after SD; bars=standard deviation. (C) Typical example (subject 3) of the effects of sleep deprivation on inhibitory $(3 \mathrm{~ms})$ and facilitatory $(16 \mathrm{~ms})$ interstimulus intervals. Each tracing represents the average of eight control (upper tracing) and eight conditioned (lower tracing) motor evoked potentials

analysis time $100 \mathrm{~ms}$ ), after 16 consecutive stimuli in the relaxed $\mathrm{FDI}^{10}$; (2) active threshold (aT), the same as above but during $10 \%$ of maximum voluntary contraction (MVC) of the target muscle; (3) duration of the silent period (sensitivity $5 \mathrm{mV} /$ division, analysis time $500 \mathrm{~ms}$ ). This represented the period of suppression of the EMG activity produced by a magnetic shock set as $1.5 \times \mathrm{rT}$ in the preactivated right first dorsal interosseous muscle ( $10 \%$ of MVC). The duration of the silent period went from the stimulus artefact to the reappearing of the EMG activity in eight superimposed, non-rectified trials. A sphygmomanometer was used for the monitoring of the appropriate level of muscle preactivation. The subjects squeezed the partially inflated cuff between their extended first and second finger. The amount of contraction was expressed as $\mathrm{mm} \mathrm{Hg}$. The inhibitory and facilitatory interstimulus intervals (ISIs) were studied with the paired pulse technique ${ }^{8}$. The intensity of the conditioning stimulus was $0.8 \times \mathrm{rT}$; and the intensity of the test stimulus was $1.2 \times r \mathrm{rT}$. The second was adjusted to evoke test MEPs $0.5-1 \mathrm{mV}$ in amplitude. Attention was focused on the two most inhibitory ( 2 and $3 \mathrm{~ms}$ ) and facilitatory (14 and $16 \mathrm{~ms}$ ) ISIs (sensitivity $0.5 \mathrm{mV} /$ division; analysis time $100 \mathrm{~ms}$ ). For each ISI, we recorded eight control and eight conditioned MEPs in a random order. The effect of the conditioning was expressed as a ratio of the averaged conditioned MEP at each ISI to the averaged control MEP. Averaged MEPs were from non-rectified EMG signals. Inhibition occurred when the ratio was less then one, facilitation when it was greater then one. For determination of the relaxed threshold and the paired pulse curve, full relaxation of the interosseous muscle was necessary. To facilitate this, subjects were given audio feedback of the background activity in the high gain EMG signal through a loudspeaker. If an undue EMG background appeared in these experiments, the given signal was discarded. To test the peripheral and spinal motor excitability the maximum $\mathrm{M}$ wave (Mmax) and $\mathrm{F}$ wave amplitude was determined by supramaximal stimulation of the ulnar nerve at the wrist in the resting condition. Sixteen responses were collected for each test.

Sleep deprivation consisted of a period of total sleep loss of at least 24 hours, and was performed according to the routine suggested by Naitoh and Dement. ${ }^{11}$ To avoid any hypothetical "order effect", the baseline TMS study was performed before the one that followed sleep deprivation in five subjects, whereas the sequence was reversed in the remaining three. In this last group, at least three sleep/wake cycles represented the recovery from sleep deprivation, which was held sufficient for the "baseline" test. In theory, somnolence might interfere with the degree of subject cooperation during the experiments. Thus, the level of sleepiness before and after sleep deprivation was determined with the Stanford sleepiness scale. ${ }^{12}$

For statistical analysis the SPSS program (release 8.0.0) for Windows 95 was used. Data on $\mathrm{rT}$, aT, and the silent period were compared by means of Mann-Whitney $U$ tests, with a Bonferroni correction of $\mathrm{p}$ values. For the paired pulse curve, a repeated measure analysis of variance (ANOVA) model was used, where the main factor "time" included the different ISIs. The fixed factor was the sleep deprivation. Provided the $F$ was significant $(p<0.05)$, paired $t$ tests with a Bonferroni correction of $\mathrm{p}$ values were used to compare the single ISIs.

\section{Results}

In all subjects, the average score on the Stanford sleepiness scale ${ }^{12}$ was equal to 1 before, and equal to 3.5 after the sleep deprivation. In other words, somnolent subjects were still sufficiently alert and able to follow the 
experimental instructions. After sleep deprivation, the threshold to stimulation (in the resting and active muscle), as well as the silent period did not change significantly. Moreover, there were no significant changes in the Mmax and F wave amplitude. Subsequently the intracortical inhibition and facilitation index were estimated, as previously done by others. ${ }^{13}$ The inhibitory ratios for the interstimulus intervals of 2 and $3 \mathrm{~ms}$, and the facilitatory ratios for intervals of 14 and $16 \mathrm{~ms}$ were combined. After the sleep deprivation, there was a significant $(F(1,7)=5.59, \mathrm{p}<0.05)$ reduction of both inhibition (Bonferroni $p=0.025$ ) and facilitation (Bonferroni $\mathrm{p}=0.019$, fig $1 \mathrm{~A}$ ). Next, the effects of the sleep deprivation on the single ISIs (fig 1 B) were analysed. The inhibitory effect was more evident at ISI $3 \mathrm{~ms}$ (Bonferroni $\mathrm{p}=0.05$ ), whereas facilitation had a maximum at ISI 16 ms (Bonferroni $\mathrm{p}=0.017$ ). Figure 1 (panel C) shows a typical example of the MEP tracings in a paired pulse experiment.

\section{Discussion}

Sleep deprivation induced changes in some of the TMS variables studied, which we attribute to alterations of the primary motor cortex excitability. In fact, indexes of spinal ( $F$ waves) or neuromuscular ( $M$ waves) excitability remained unaltered. Changes in TMS, however, did not affect the motor threshold or the silent period. Motor threshold most likely reflects axon membrane excitability, because drugs acting on voltage and frequency dependent sodium and calcium channels modulate it. ${ }^{13}$ Thus we assume that sleep deprivation had no direct effect on the membrane of the nerve cells presynaptic to the corticospinal neuron; these largely represent the primary excitation site of TMS. ${ }^{10}$ The silent period has a more complex, and perhaps obscure, pathophysiology, ${ }^{14}$ yet sleep deprivation seemed to alter none of its many putative generators. The sensitive variable was the intracortical inhibition and facilitation curve, both phases of which were depressed. The first (1-5 ms) phase, inhibition, is thought to rely on a different interneuron circuit from the second one (7-16 ms) - that is, facilitation. ${ }^{9}$ Both arise from the cerebral cortex, as the conditioning pulses are too weak to activate the corticospinal tract. ${ }^{8}$ Some authors made inferences to the transmitters implied in these phenomena. Antiepileptic GABAergic drugs were able to reduce facilitation, and, to some extent, enhance inhibition. ${ }^{13}$ NMDA-receptor antagonists ${ }^{15}$ and dopamine agonists were attributed a similar effect, whereas the reverse was true for dopamine antagonists. ${ }^{16}$ On this basis, paired pulse TMS was proposed as an assay of both excitability and pharmacology of the interneuronal circuitry in the primary motor cortex. ${ }^{13}{ }^{17}$ In general, most of the conditions studied showed an inverse correlation between inhibition and facilitation. If the second decreased, the first increased, and vice versa. A partial exception to this rule might be the effects of vigabatrin, ${ }^{13}$ a typical GABAergic drug that reduced facilitation without affecting inhibition, or the serotonergic $5 \mathrm{HT}_{1 \mathrm{~B} / 1 \mathrm{D}}$ agonist zolmitriptan, ${ }^{18}$ which reduced inhibition leaving facilitation unaffected. In general, the intimate pharmacological nature of the paired pulse effects seems to need further studies. In our present findings, however, loss of inhibition was unexpectedly coupled with reduction of facilitation. The coexistence of such apparently opposing phenomena is difficult to interpret. In theory, proepileptogenic and antiepileptogenic effects would seem to cancel each other. To us, it may be more useful to note that sleep deprivation was associated with a general hypoactivity of cortical area 4 interneurons, reflected by the flattening of the paired pulse curve. Besides, excess excitation, and defective but also excessive inhibition, interact in a very complex manner to predispose the cortex to epileptiform discharges. ${ }^{19}$ Thus, we cannot exclude the possibility that our findings might be compatible with an "activating" net effect within the cortex.

As our method explored the primary motor cortex, the relevance of our data to those epileptiform activities which might affect the brain with a different topography may be questioned. Yet, area 4 excitability was found altered in various epileptic syndromes, not only generalise ${ }^{20}$ but also partial (for example, with temporal foci). ${ }^{21}$ In the last condition, loss of intracortical inhibition is related to a more severe EEG and clinical picture. ${ }^{21}$ Thus, area 4 physiology - in the epilepsy field - proved sensitive to phenomena that exceed its boundaries.

In conclusion, TMS disclosed some subtle changes in normal cortical physiology, which may serve as a model for studying the "activating" effects of sleep deprivation in patients with epilepsy.

This work was presented in abstract form at the 10th European Congress of Clinical Neurophysiology. Lyon, France: 26-30 August, 2000

1 Gastaut H, Tassinari CA. Triggering mechanisms in epilepsy. The electroclinical point of view. Epilepsia 1966;7:85-138.

2 Scollo-Lavizzari G, Scollo-Lavizzari GR. Sleep, sleep deprivation, photosensitivity, and epilepsy. Eur Neurol 1974;11: $1-21$

3 Berti-Ceroni G, Sabattini L, Gambi D, et al. Effetti della "sleep deprivation" in epilettici. Riv Neurol 1967;37:30520.

4 Fountain NB, Kim JS, Lee SI. Sleep deprivation activates epileptiform discharges independent of the activating effects of sleep. F Clin Neurophysiol 1998;15:69-75.

5 Cohen HB, Dement WC. Sleep: changes in threshold to electroconvulsive shock in rats after deprivation of "paradoxical" phase. Science 1965;150:1318-19.

6 Shouse MN. Sleep deprivation increases thalamocortical excitability in the somatomotor pathways, especially during seizure-prone sleep or awakening states in the feline seizure models. Exp Neurol 1988;99:664-77.

7 Rodin E. Sleep deprivation and epileptological implications. In Degen R, Rodin EA, eds. Epilepsy, sleep and sleep depIn Degen R, Rodin EA, eds. Epilepsy, sleep a
rivation. Epilepsy Res 1991;(suppl 2):265-73.

8 Kujirai T, Caramia MD, Rothwell JC, et al. Corticocortical Kujirai T, Caramia MD, Rothwell JC, et al. Corticocortical
inhibition in the human motor cortex. $\mathcal{f}$ Physiol (Lond) 1993;471:501-19.

9 Ziemann U, Rothwell JC, Ridding MC. Interaction between intracortical inhibition and facilitation in human motor cortex. F Physiol (Lond) 1996;496:873-81.

10 Rossini PM, Barker AT, Berardelli A, et al. Non-invasive electrical and magnetic stimulation of the brain, spinal cord and roots: basic principles and procedures for routine clinical application. Report of an IFCN Committee. Electroencephalogr Clin Neurophysiol 1994;91:79-82.

11 Naitoh P, Dement W. Sleep deprivation in humans. In: Remond A, ed. Handbook of electroencephalography and clinical neurophysiology. Amsterdam: Elsevier, 1976:146-51.

12 Hoddes E, Zarcone V, Smythe H, et al. Quantification of Hoddes E, Zarcone V, Smythe H, et al. Quantification of
sleepiness: a new approach. Psychobiology 1973;10:431-6. 13 Ziemann U, Lonnecker S, Steinhoff BJ, et al. Effects of antiepileptic drugs on motor cortex excitability in humans: a transcranial magnetic stimulation study. Ann Neurol 1996; 40:367-78. 
14 Cantello R, Gianelli M, Civardi C, et al. Magnetic brain stimulation: the silent period after the motor evoked potenstimulation: the silent period after

15 Ziemann U, Chen R, Cohen LG, et al. Dextromethorphan decreases the excitability of the human motor cortex. Neurology 1998;51:1320-4.

16 Ziemann U, Tergau F, Bruns D, et al. Changes in human motor cortex excitability induced by dopaminergic and anti-dopaminergic drugs. Electroencephalogr Clin Neurophysiol 1997;105:430-7.

17 Ziemann U, Steinhoff BJ Tergau F, et al. Transcranial magnetic stimulation: its current role in epilepsy research. Epilepsy Res 1998;30:11-30.
18 Werhahn KJ, Förderreuther S, Straube A. Effects of the serotonin $1 \mathrm{~B} / 1 \mathrm{D}$ receptor agonist zolmitriptan on motor cortical excitability in humans. Neurology 1998;51:896-8.

19 Engel J Jr. Excitation and inhibition in epilepsy. Can f Neurol Sci 1996;23:167-74.

20 Caramia MD, Gigli G, Iani C, et al. Distinguishing forms of generalized epilepsy using magnetic brain stimulation. Electroencephalogr Clin Neurophysiol 1996;98:14-19.

21 Cantello R, Civardi C, Cavalli A, et al. Cortical excitability in cryptogenic localization-related epilepsy: interictal transcranial magnetic stimulation studies. Epilepsia 2000;41: 694-704

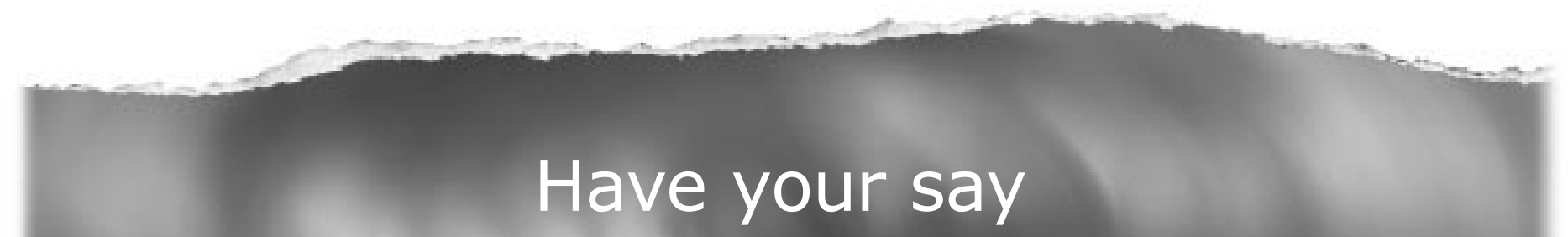

\section{eLetters}

If you wish to comment on any article published in the Journal of Neurology, Neurosurgery, and Psychiatry you can send an eLetter using the eletters link at the beginning of each article. Your response will be posted on Journal of Neurology, Neurosurgery, and Psychiatry online within a few days of receipt (subject to editorial screening).

\section{www.jnnp.com}

